\title{
Mediastinal lymph node metastasis of renal cell carcinoma: A case report
}

\author{
KUNIHIKO MIYAZAKI ${ }^{1}$, SHINYA SATO ${ }^{1}$, TAKAHIDE KODAMA ${ }^{1}$, KOICHI KURISHIMA $^{2}$, \\ HIROAKI SATOH ${ }^{3}$ and NOBUYUKI HIZAWA ${ }^{4}$ \\ ${ }^{1}$ Division of Respiratory Medicine, Ryugasaki Saiseikai Hospital, Ryugasaki, Ibaraki 301-0854; \\ ${ }^{2}$ Division of Respiratory Medicine, Tsukuba Medical Center Hospital, Tsukuba, Ibaraki 305-8558; \\ ${ }^{3}$ Division of Respiratory Medicine, Mito Medical Center, University of Tsukuba, Mito, Ibaraki 310-0015; \\ ${ }^{4}$ Division of Respiratory Medicine, Faculty of Medicine, University of Tsukuba, Tsukuba, Ibaraki 305-8575, Japan
}

Received December 8, 2014; Accepted December 11, 2015

DOI: $10.3892 / \mathrm{ol} .2016 .4090$

\begin{abstract}
Renal cell carcinoma (RCC) may metastasize to mediastinal lymph nodes without any abdominal lymph node involvement. The present study describes an autopsy-proven case of RCC presenting with a large mediastinal mass; the case had been previously misdiagnosed as small cell lung carcinoma due to imaging analysis results, an elevated serum level of neuron-specific enolase and the presence of small atypical cells with a high nuclear/cytoplasmic ratio. Despite RCC occurrence being rare, it should be considered in the differential diagnosis, particularly when a mass located in the kidneys presents with metastases to the mediastinal lymph nodes, even if there is no involvement of the abdominal lymph nodes and the primary lesion is of a small size.
\end{abstract}

\section{Introduction}

Renal cell carcinoma (RCC) accounts for $95 \%$ of renal neoplasms and $\sim 3 \%$ of adult malignancies (1). In the United States, $\sim 61,560$ patients with RCC were identified and 14,080 patients were predicted to have succumbed to the disease in 2015 (2). Generally, patients with RCC present with gross hematuria, flank pain and a palpable mass; however, these symptoms are not always present. RCC is a heterogeneous disease and several histological subtypes have been categorized according to the 2004 World Health Organization (WHO) renal tumor classification (3). The mortality rate of patients with RCC depends on the histological subtype and the clinical stage of the tumor. Since RCC has a hemorrhagic tendency, biopsy of the tumor is disputed (4). A diagnosis of RCC is provided according to the results observed using imaging methodologies, including

Correspondence to: Professor Hiroaki Satoh, Division of Respiratory Medicine, Mito Medical Center, University of Tsukuba, 3-2-7 Miya-machi, Mito, Ibaraki 310-0015, Japan

E-mail: hirosato@md.tsukuba.ac.jp

Key words: mediastinal lymph node metastasis, renal cell carcinoma, clear cell carcinoma computed tomography (CT), and a diagnosis of RCC is confirmed by the surgical removal of the tumor en bloc. The present study reports the case of a patient who presented with a large, right mediastinal mass appearing to mimic small cell lung cancer (SCLC); however, following autopsy examination, the diagnosis was confirmed as RCC.

\section{Case report}

A 56-year-old male presented at the Ryugasaki Saiseikai Hospital (Ryugasaki, Japan) in September 2011 with a dry cough that had persisted for 3 months. Physical examination identified decreased breath sounds at the right hemithorax. Laboratory evaluations were undertaken and included urinalysis and testing of blood urea nitrogen, creatinine and serum calcium concentrations. All laboratory analyses were normal, with the exception of an increased concentration of white blood cells $(12,100$ cells $/ \mu 1$; normal range, 3,900-9,800 cells $/ \mu \mathrm{l})$, C-reactive protein $(12.9 \mathrm{mg} / \mathrm{dl}$; normal range, $0.0-0.3 \mathrm{mg} / \mathrm{dl}$ ) and serum neuron-specific enolase (NSE; $50.0 \mathrm{ng} / \mathrm{ml}$; normal range, 0.0-16.3 ng/ml). Following a CT scan of the chest, a large mass was detected at the right mediastinum, extending directly to the heart, with the presence of pericardial fluid (Fig. 1). Pleural fluid was identified in the right hemithorax, but no abnormalities were observed in the lungs and the abdominal lymph nodes were not swollen. However, a small nodule was detected in the upper pole of the right kidney. A bronchoscopy was performed and a mass was identified in the right middle lobe bronchus. A specimen of the lesion was taken for cytological examination. Analysis of the lesion demonstrated the presence of small atypical cells with a high nuclear/cytoplasmic $(\mathrm{N} / \mathrm{C})$ ratio; the mass was subsequently diagnosed as SCLC. Additionally, the patient presented with a metastatic lesion in the brain, and it was evaluated as an extension of the disease. The clinical condition of the patient rapidly deteriorated, leading to the initiation of carboplatin-containing chemotherapy (one course of carboplatin, area under the curve $=5$, every day for 28 days; one course of etoposide, $100 \mathrm{mg} / \mathrm{m}^{2}$, every day for 28 days) subsequent to the bronchoscopy. Tentative pleural fluid of the right hemithorax decreased in response to chemotherapy, however, 


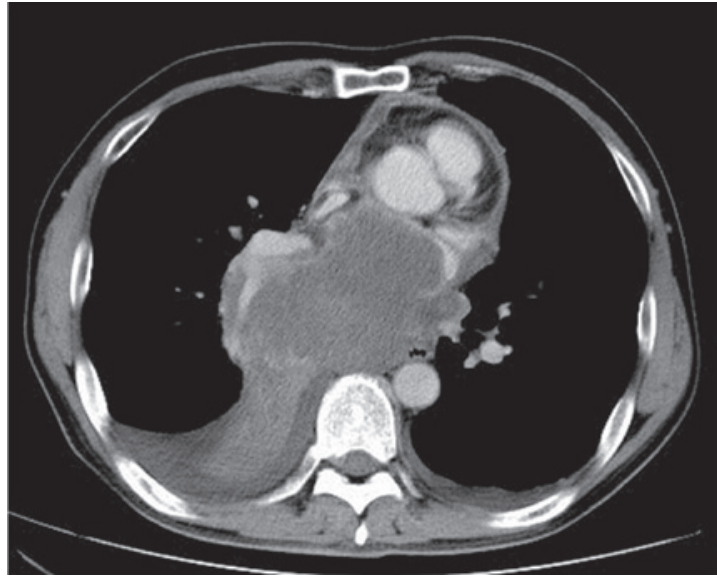

Figure 1. Computed tomography scan demonstrating a large mass located at the right mediastinum, extending directly to the heart, with the presence of pericardial fluid.

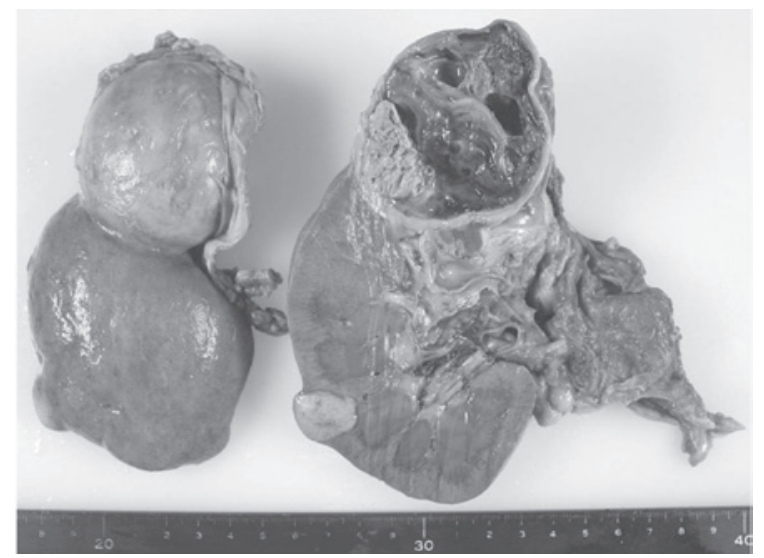

Figure 2. Autopsy examination identified two tumors in the right kidney; one measured $20 \times 20 \times 18 \mathrm{~mm}$ in diameter and was located in the lower pole of the kidney (left of image), and the second tumor measured $63 \times 55 \times 50 \mathrm{~mm}$ in diameter and was located in the upper pole of the kidney (right of image).

the respiratory condition of the patient declined. Following two courses of chemotherapy, a routine evaluation observed enlargement of the mediastinal and renal masses. Furthermore, CT (LightSpeed VCT 64; GE Healthcare Healthcare Bio-Sciences, Pittsburgh, PA, USA) identified a novel lesion located in the head of the pancreas. The patient succumbed to the disease shortly after the performance of CT.

Autopsy examination demonstrated a large right mediastinal lymph node metastasis invading the right lung, the ipsilateral mediastinum, the pericardium and the heart. However, no association with the abdominal lymph nodes was observed. Despite no tumor thrombus being identified in the renal vein and inferior vena cava, two tumors in the right kidney were detected; one measured $63 \times 55 \times 50 \mathrm{~mm}$ in diameter and was located in the upper pole of the kidney, and the second tumor measured 20x20x18 mm in diameter and was located in the lower pole of the kidney (Fig. 2). Microscopically, the two tumors were established as the clear cell RCC subtype, although cell-cell adhesion was loose in the lower tumor of the kidney (Fig. 3). Immunohistochemical staining was performed using the following antibodies at a 1:100 dilution: Rabbit monoclonal anti-human cluster of differentiation (CD)10 (cat no. M3670),
A

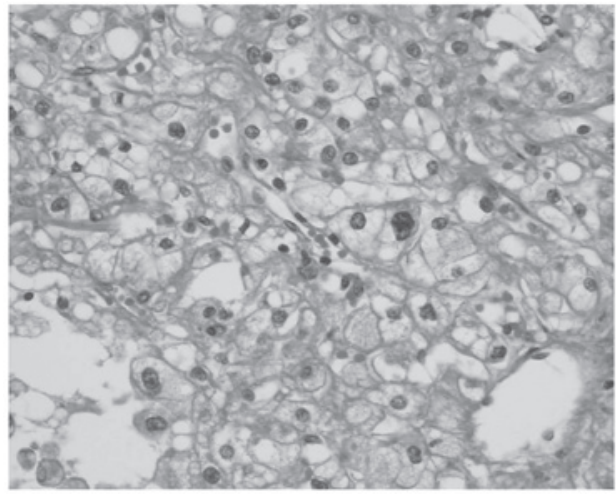

$\mathbf{B}$

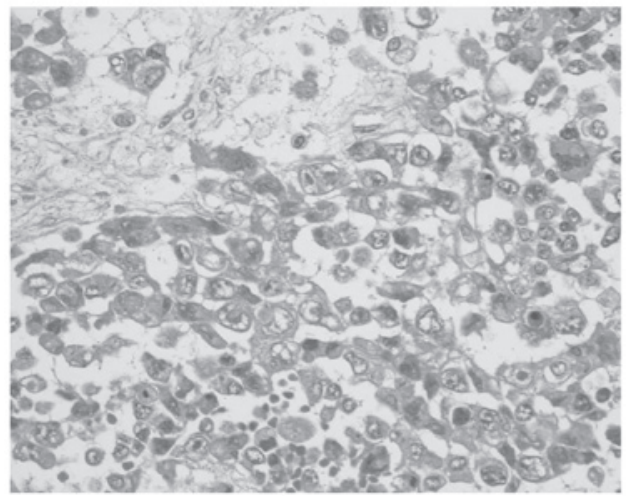

Figure 3. Microscopic examination of two kidney tumors. (A) A tumor located at the upper pole and (B) a tumor at the lower pole of the right kidney. Each was confirmed as clear cell RCC, although cell-cell adhesion was loose in the lower tumor of the kidney. Hematoxylin and eosin staining; magnification, $\mathrm{x} 200$.

mouse monoclonal anti-human CD15 (cat no. cd15-250), rabbit monoclonal anti-human vimentin (cat no. OPPA01225) and mouse monoclonal anti-human CD7 (cat no. ABIN1384047), which were all purchased from Funakoshi, Co., Ltd. (Tokyo, Japan). Immunohistochemical staining was observed to be positive for CD10, CD15 and vimentin, and was negative for CD7. The same cancer cells were identified in the metastatic tumors in the pancreas head, the mediastinum and the hilar lymph nodes.

Written informed patient consent was obtained from the family of the patient for the publication of this study.

\section{Discussion}

RCC is a heterogeneous disease and 12 distinct histological subtypes have been categorized in the 2004 WHO classification of renal cell tumors (3). Clear cell RCC accounts for $\sim 75 \%$ of renal cell tumors; the other subtypes are typically summarized under the term non-clear cell RCC $(5,6)$. RCC typically develops during adulthood, affecting individuals $>40$ years of age, with the incidence peaking during the sixth and seventh decades of life. The classic triad of symptoms consists of gross hematuria, flank pain and a palpable mass (7). RCC generally presents with one or more of these features. A key prognostic factor is the clinical stage of the disease at the time of diagnosis. Metastatic RCC is present in $20-30 \%$ of patients at diagnosis, with the majority of these demonstrating multiple metastatic sites $(8,9)$. It has been established that RCC can invade the renal vein and extend to the inferior vena cava. Furthermore, RCC metastasizes through hematogenous and lymphogenous techniques, and 
may also metastasize through the Batson paravertebral venous plexus, which communicates with the azygos or hemiazygos veins $(10,11)$. The lungs, bones, liver, lymph nodes and mediastinum are frequent metastatic sites (12). Among these, the lungs are understood to be the most common site of metastasis, with pleural dissemination also reported (13). Koç et al (14) described the case of a 14-year-old male who presented with clear cell RCC, which had metastasized to the mediastinal lymph nodes; a large mediastinal metastatic mass, without any association with the abdominal lymph nodes, was the predominant finding of the case, as was similarly observed in the present case. In the current study, the key observations were mediastinal lymph node metastasis and respiratory distress, which were also observed in the case reported by Koç et al (14). In the present case, at the time of autopsy, clear cell RCC of the kidney (with tumors measuring 60 and $20 \mathrm{~mm}$ in diameter) was confirmed, and an identical diagnosis was determined for the lesions observed in the mediastinal lymph nodes. As there was no evidence of a tumor thrombus in the renal vein and inferior vena cava, the possibility of hematogenous metastasis appeared to be unlikely.

Several studies have noted elevated serum levels of NSE in cases of RCC $(15,16)$, as was observed in the current case. NSE is a neuroendocrine marker and is established as a reliable tumor marker of SCLC (17). Yaman et al (16) studied serum NSE in $25 \mathrm{RCC}$ patients and reported that it may serve as a useful marker in the surveillance and evaluation of the disease. Rasmuson et al (15) evaluated chromogranin A and NSE as neuroendocrine markers in 200 patients with RCC and concluded that serum NSE was a significant predictor of prognosis. In the present case, in addition to enlarged hilar and mediastinal masses, the observation of elevated serum NSE levels and positive immunohistochemical staining of neuroendocrine markers, including chromogranin A, suggested a diagnosis of SCLC. However, the poor patient response to platinum-containing chemotherapy did not correspond with SCLC. Autopsy findings demonstrated that the initial diagnosis of SCLC was incorrect. A large mass presenting with elevated serum NSE and small atypical cells with a high N/C ratio had been obtained during a bronchoscopy and suggested that a primary SCLC lesion may be located adjacent to the mediastinum, forming a large mass with the metastatic mediastinal lymph nodes. Metastases to the lungs, pleura and mediastinal lymph nodes from primary RCC lesions are not rare; however, renal metastasis from a primary SCLC lesion is rare (18).

The prognosis of patients with metastatic RCC remains poor, since a key prognostic factor in RCC is the clinical stage of the disease (19). Several targeted therapy drugs, such as sunitinib, sorafenib, bevacizumab and temsirolimus, have been approved for use in patients with RCC (20). However, in addition to cytotoxic drugs, molecular targeting agents, including mammalian target of rapamycin inhibitors and tyrosine kinase receptors, have recently been introduced for the treatment of patients with RCC, and a survival benefit has been demonstrated in certain clinical trials $(21,22)$.

In conclusion, despite the occurrence of RCC being rare, it should be considered in the differential diagnosis, particularly when a mass located in the kidneys presents with metastases to the mediastinal lymph nodes and elevated NSE serum levels, even if there is no involvement of the abdominal lymph nodes and the primary lesion is of a small size.

\section{References}

1. Jemal A, Siegel R, Ward E, Hao Y, Xu J and Thun MJ: Cancer statistics, 2009. CA Cancer J Clin 59: 225-249, 2009.

2. American Cancer Society: Cancer Facts and Figures 2015. American Cancer Society, Inc., Atlanta, GA, 2015.

3. Lopez-Beltran A, Scarpelli M, Montironi R and Kirkali Z: 2004 WHO classification of the renal tumors of the adults. Eur Urol 49: 798-805, 2006

4. Lee HM, Kang HJ and Lee SH: Metastatic renal cell carcinoma presenting as epistaxis. Eur Arch Otorhinolaryngol 262: 69-71, 2005.

5. Aronson DC, Medary I, Finlay JL, Herr HW, Exelby PR and La Quaglia MP: Renal cell carcinoma in childhood and adolescence: A retrospective survey for prognostic factors in 22 cases. J Pediatr Surg 31: 183-186, 1996.

6. Eckschlager T and Kodet R: Renal cell carcinoma in children: A single institution's experience. Med Pediatr Oncol 23: 36-39, 1994.

7. Jayson M and Sanders H: Increased incidence of serendipitously discovered renal cell carcinoma. Urology 51: 203-205, 1998.

8. Geller E, Smergel EM and Lowry PA: Renal neoplasms of childhood. Radiol Clin North Am 35: 1391-1413, 1997.

9. Flanigan RC and Yonover PM: The role of radical nephrectomy in metastatic renal cell carcinoma. Semin Urol Oncol 19: 98-102, 2001.

10. LaBan MM, Wilkins JC, Szappanyos B, Shetty AN and Wang AM: Paravertebral plexus of veins (Batson's) - potential route of paraspinal muscle metastases as imaged by magnetic venous angiography. A commentary. Am J Phys Med Rehabil 76: 517-519, 1997.

11. Kuba H, Sato N, Uchiyama A, Nakafusa Y, Mibu R, Yoshida K, Kuroiwa K and Tanaka M: Mediastinal lymph node metastasis of colon cancer: Report of a case. Surg Today 29: 375-377, 1999.

12. Pratt CB and Douglass EC: Management of the less common cancers of childhood. In: Principles and Practice of Pediatric Oncology. Pizzo PA and Poplack DG (eds). Lippincott, Philadelphia, pp913-938, 1993.

13. Davion S, Rohan S, Nayar R and Kulesza P: Metastatic chromophobe renal cell carcinoma in pleural fluid cytology: Review of literature and report of a case. Diagn Cytopathol 40: 826-829, 2012.

14. Koc M, Polat P, Erem T, Büyükavci M, Ozbey I, Gündogdu C and Suma S: Quiz case of the month. Diagnosis: Clear-cell renal cell carcinoma (RCC) with metastasis to lung, mediastinal and abdominal lymph nodes and bones. Eur Radiol 9: 1935-1936, 1999.

15. Rasmuson T, Grankvist $\mathrm{K}$, Roos $\mathrm{G}$ and Ljungberg B: Neuroendocrine differentiation in renal cell carcinoma - evaluation of chromogranin A and neuron-specific enolase. Acta Oncol 38: 623-628, 1999.

16. Yaman O, Baltaci S, Arikan N, Ozdiler E, Göğüs $O$ and Müftüoğlu YZ: Serum neuron specific enolase: Can it be a tumour marker for renal cell carcinoma? Int Urol Nephrol 28: 207-210, 1996

17. Niklinski J and Furman M: Clinical tumour markers in lung cancer. Eur J Cancer Prev 4: 129-138, 1995.

18. Burgess RE, Burgess VF and Dibella NJ: Brain metastases in small cell carcinoma of the lung. JAMA 242: 2084-2086, 1979.

19. Santoni M, De Tursi M, Felici A, Lo Re G, Ricotta R, Ruggeri EM, Sabbatini R, Santini D, Vaccaro V and Milella M: Management of metastatic renal cell carcinoma patients with poor-risk features: Current status and future perspectives. Expert Rev Anticancer Ther 13: 697-709, 2013.

20. Minguet J, Smith KH, Bramlage CP and Bramlage P: Targeted therapies for treatment of renal cell carcinoma: Recent advances and future perspectives. Cancer Chemother Pharmacol 76: 219-233, 2015.

21. Diamond E, Molina AM, Carbonaro M, Akhtar NH, Giannakakou P, Tagawa ST and Nanus DM: Cytotoxic chemotherapy in the treatment of advanced renal cell carcinoma in the era of targeted therapy. Crit Rev Oncol Hematol 96: 518-526, 2015.

22. Iacovelli R, Alesini D, Palazzo A, Trenta P, Santoni M, De Marchis L, Cascinu S, Naso G and Cortesi E: Targeted therapies and complete responses in first line treatment of metastatic renal cell carcinoma. A meta-analysis of published trials. Cancer Treat Rev 40: 271-275, 2014. 